\title{
IMAGEN DE LA LICENCIATURA EN ADMINISTRACIÓN (UNR) EN AQUELLOS ESTUDIANTES DE CIENCIAS ECONÓMICAS QUE NO LA ELIGIERON
}

\author{
Oviedo, Raúl Ricardo \\ Racca, Adriana Patricia \\ Suñer, María Natalia \\ Fior, Marina \\ Ford, Jairo Jesús
}

\section{RESUMEN:}

El presente artículo aborda la imagen que tienen de la Licenciatura en Administración los alumnos de las demás carreras de Ciencias Económicas, en torno a su perfil profesional, competencias específicas, ámbito de desarrollo profesional entre otras cuestiones.

Luego de realizar un relevamiento de corte cuantitativo se obtuvo como resultado que, con sus matices, tanto los estudiantes de Contador Público como de Licenciatura en Economía coinciden en la mayoría de sus respuestas en lo que respecta a la representación de la Licenciatura en Administración.

PALABRAS CLAVE: Alumnos; Carrera Universitaria; Ciencias Económicas; Contador Público; Licenciatura en Administración; Licenciatura en Economía

\section{ABSTRACT:}

This article deals with the image of the Bachelor's Degree in Administration that students of other careers in Economics have, around their professional profile, specific competencies, professional development area among other issues.

After conducting a quantitative cut-off survey, the result was that, with their nuances, both Public Accountant and Bachelor's Degree in Economics students agree on most of their answers regarding the representation of the Bachelor's Degree in Administration.

Keywords: Students; University Career; Economics; Public Accountant; Bachelor's Degree in Administration; Bachelor's Degree in Economics

\section{INTRODUCCIÓN}

A través del tiempo, las carreras que forman parte de la formación del profesional en Ciencias Económicas van evolucionando y reinventándose como consecuencia de estar inmersas en un contexto dinámico y cambiante.

Los trabajos y relaciones laborales exigen nuevas formas de vincularse y desempeñarse y, por lo tanto, las exigencias de los mismos sufren cambios que suelen ser plasmados en las 
ofertas educativas de las universidades para la formación de los profesionales que ejecutarán esos empleos.

Sin embargo, la dinámica de los cambios y la velocidad con la que surgen no siempre son las mismas: en el mundo laboral se solicitan habilidades y competencias nuevas, el profesional tiene sus propias expectativas, los estudiantes exigen lo suyo a la universidad y las instituciones intentan responder y modernizarse en un siglo XXI plasmado de incertidumbre.

Es así que, como docentes de la Facultad de Ciencias Económicas y Estadística, más específicamente de la Licenciatura en Administración, surgió la pregunta respecto de la expectativa profesional propia del estudiante de esa carrera en términos de la construcción de su perfil profesional y su opinión con respecto a la formación adquirida en esta Casa de Estudios.

Para complementar esa perspectiva, en el presente trabajo, se pretende indagar sobre la construcción de los estudiantes de las otras carreras universitarias que forman parte de las Ciencias Económicas (Contador Público y Licenciatura en Economía) en lo referente al perfil profesional del Licenciado en Administración, sus competencias específicas en torno a la carrera, así como sus ámbitos de desarrollo profesional, entre otras. Es importante destacar que las tres carreras universitarias que componen las Ciencias Económicas poseen en el actual plan de estudios un ciclo introductorio común de un año de duración.

El presente informe finaliza con una conclusión que plasma la información obtenida en esta investigación mostrando las opiniones, caracterizaciones y expectativas correspondientes al Licenciado en Administración suministradas por la población bajo estudio.

\section{OBJETIVOS}

El presente trabajo se propone:

- Caracterizar las representaciones de la Licenciatura en Administración presentes en los alumnos de los distintos años de las carreras de Contador Público y Licenciatura en Economía.

- Identificar el perfil profesional del Licenciado en Administración reconocido por los estudiantes de las demás carreras de Ciencias Económicas.

\section{METODOLOGÍA}

Se realizó un estudio descriptivo, de corte transversal, con un enfoque eminentemente cuantitativo.

La técnica de relevamiento aplicada es la encuesta, utilizándose en ambas poblaciones cuestionarios altamente estructurados, compuestos principalmente por preguntas cerradas y 
escalas de valoración. En esencia, las preguntas de ambos cuestionarios son las mismas, salvo sutiles diferencias en la formulación de algunas, cuando se refieren a la carrera elegida. Los cuestionarios fueron autoadministrados a través de la plataforma Survey Monkey (https://es.surveymonkey.com/).

La población es estudio son los alumnos de la carrera de Contador Público y de la Licenciatura en Economía, de segundo a quinto año, de la Universidad Nacional de Rosario. En un trabajo anterior (Oviedo, Racca, Suñer, Fior y Ford, 2021), se expusieron ya los resultados de una encuesta equivalente, con mayoría de preguntas en común, realizada a los propios alumnos de la Licenciatura en Administración.

Se partió de una base de datos provista por el Departamento Alumnado de la Facultad de Ciencias Económicas y Estadística. La muestra fue autoseleccionada; esto es, se envió la invitación a toda la población para obtener la mayor cantidad de respuestas posible.

Se obtuvo un total de 734 encuestas de Contador Público y 91 encuestas de Licenciatura en Economía.

El trabajo de campo se realizó durante el mes de septiembre de 2020.

Los datos obtenidos fueron procesados con el paquete estadístico SPSS (Statistical Package for the Social Sciences).

\section{RESULTADOS}

A continuación, se exponen los resultados de ambas encuestas, comparando las respuestas por carrera, pregunta a pregunta.

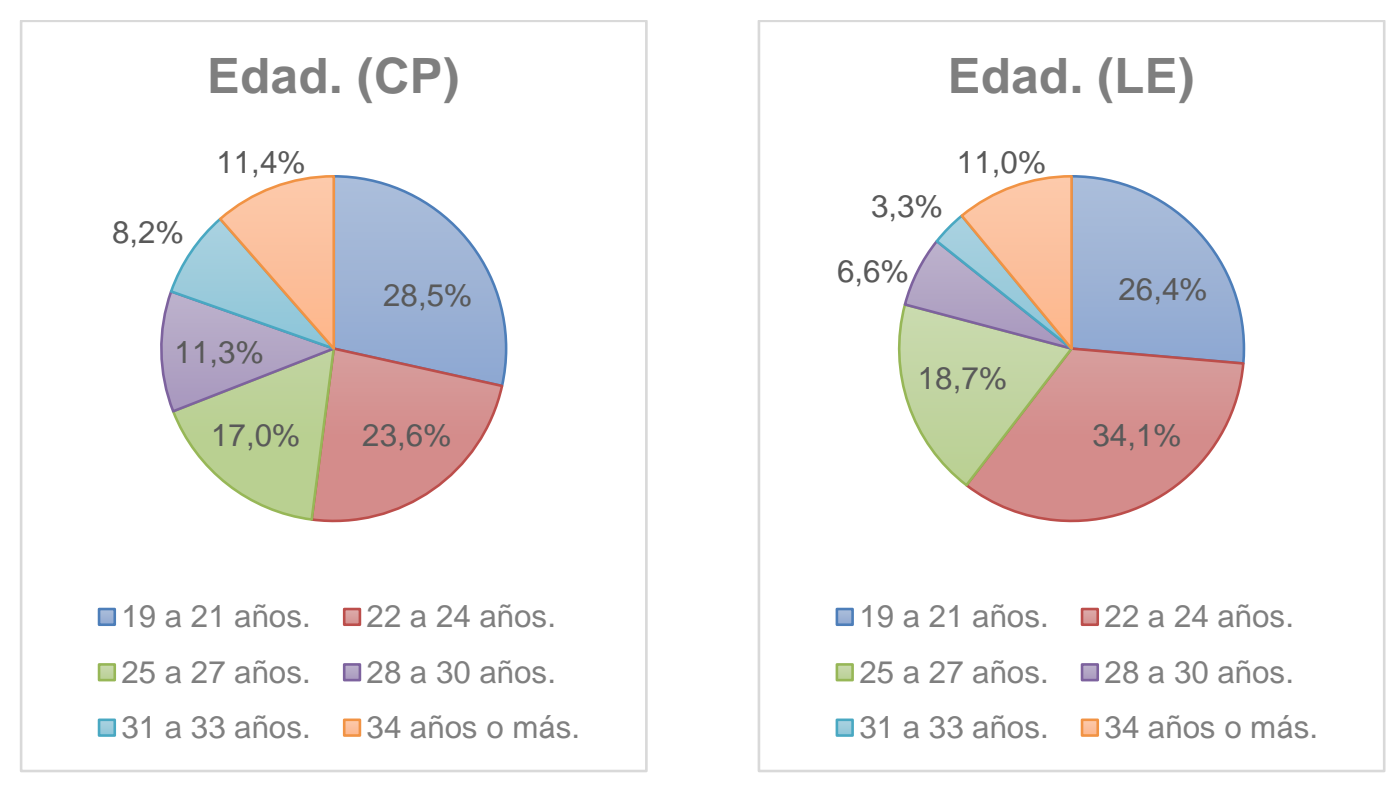

De los alumnos relevados de la carrera de Contador Público el 28,5\% tiene entre 19 y 21 años; $23,6 \%$ entre 22 y $24 ; 17 \%$ entre 25 y 27 años; $11,3 \%$ entre 28 y 30 años y $8,2 \%$ entre 31 y 33 años y $11,4 \% 34$ años o más. 
La edad promedio de los encuestados es 26,09 pero es interesante recalcar que la moda es de 20 años con una frecuencia de 78 sobre los 734 encuestados.

De los alumnos relevados de la Licenciatura en Economía el 26,4\% tiene entre 19 y 21 años; $34,1 \%$ entre 22 y $24 ; 18,7 \%$ entre 25 y 27 años; $6,6 \%$ entre 28 y 30 años y 3,3\% entre 31 y 33 años y $11 \% 34$ años o más.

La edad promedio de los encuestados es 26,16 pero es interesante recalcar que la moda es de 21 años con una frecuencia de 14 sobre los 91 encuestados.
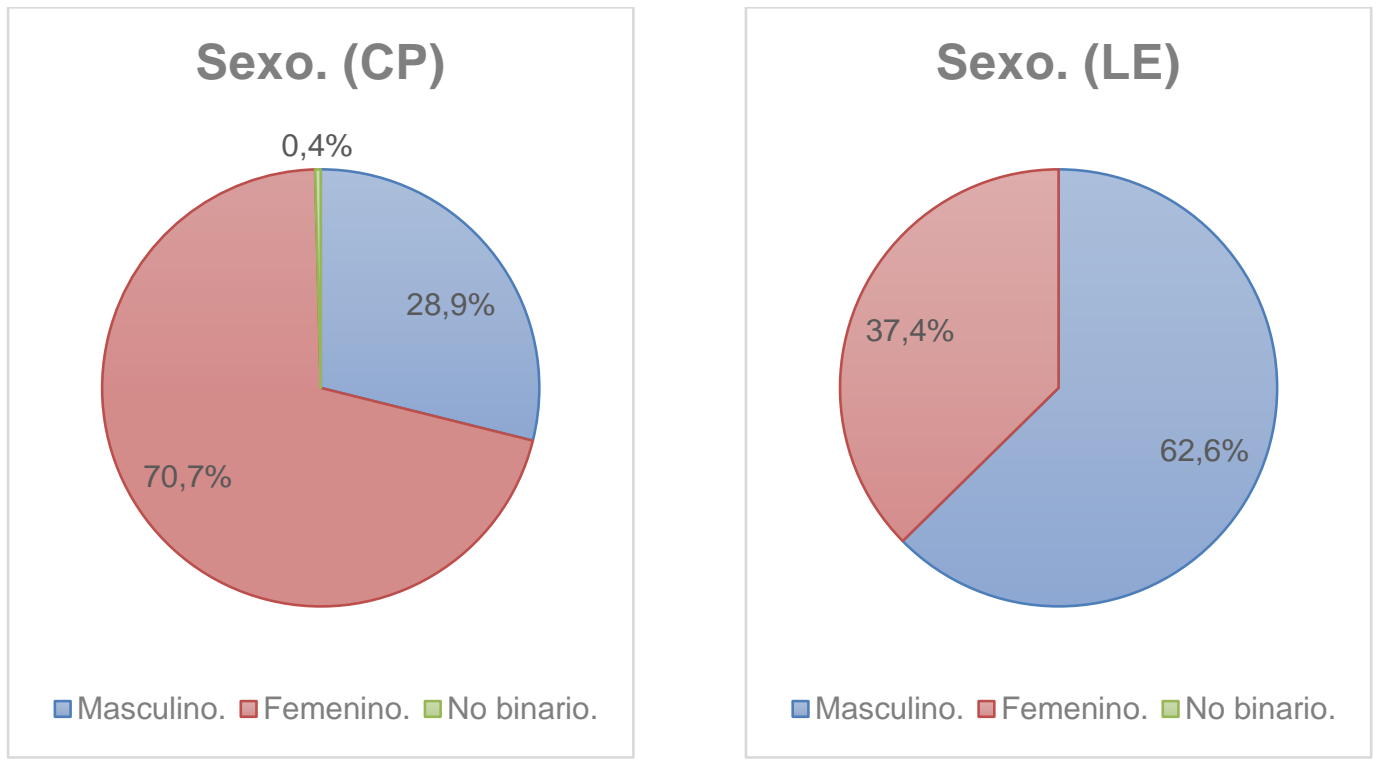

En el caso de los alumnos de Contador Público, 28,9\% se percibe de género masculino, $70,7 \%$ de género femenino y un $0,4 \%$ no binario. Esta distribución se invierte para la Licenciatura en Economía con un 37,4\% femenino y un 62,6\% masculino.
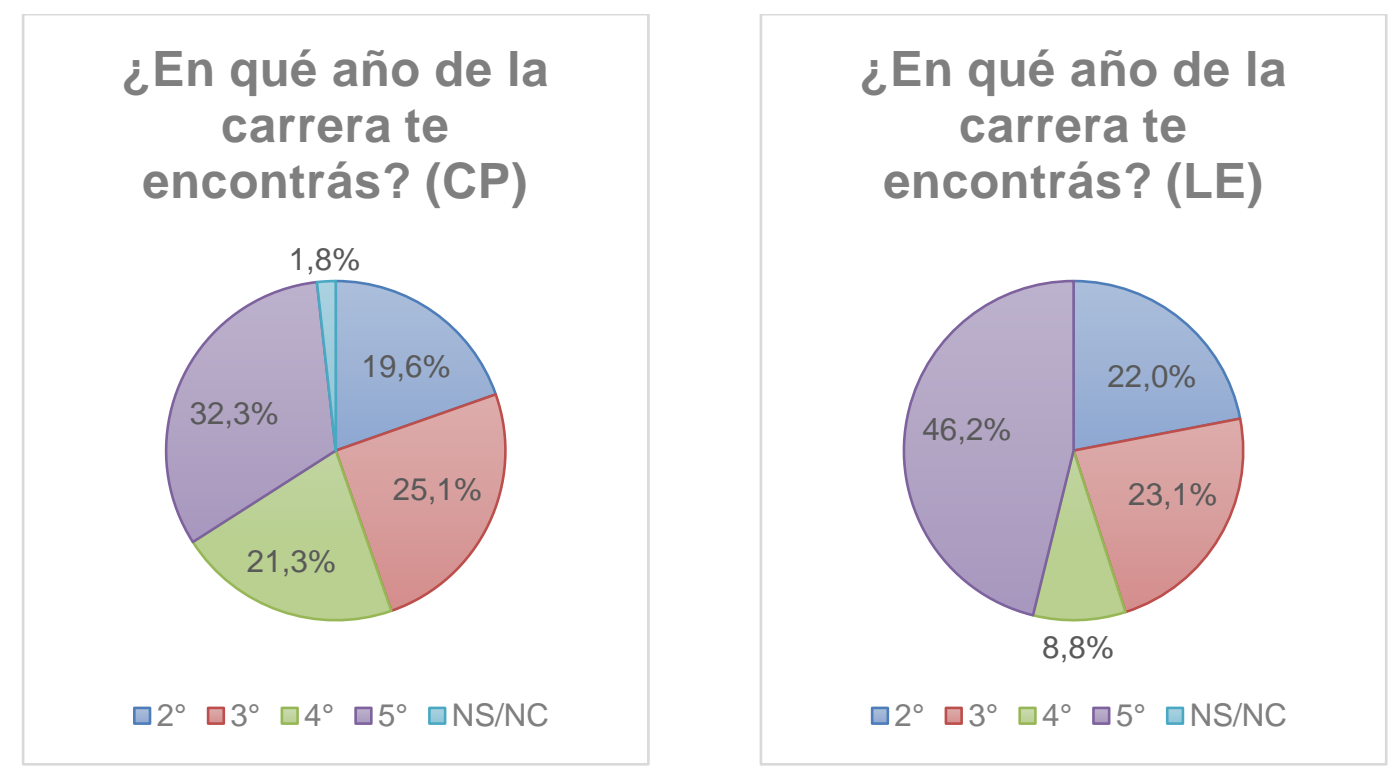

En el caso de los estudiantes de Contador encuestados, el 44,7\% están en el Ciclo Básico ( $19,6 \%$ en segundo año y $25,1 \%$ en tercero) y $53,6 \%$ en el Ciclo Superior ( $21,3 \%$ en cuarto y $32,3 \%$ en quinto año). Un $1,8 \%$ no pudo encuadrarse en un año determinado. 
En el caso de los estudiantes de Licenciatura en Economía encuestados, el 45,1\% están en el Ciclo Básico (22\% en segundo año y $23,1 \%$ en tercero) y $55 \%$ en el Ciclo Superior $(8,8 \%$ en cuarto y $46,2 \%$ en quinto año).

Vale aclarar que la encuesta se realizó a quienes ya habían elegido carrera; por lo tanto, los alumnos de primer año no están contemplados en el presente trabajo. En un trabajo anterior (Oviedo, Leda, Rubino, Said y Schellas, 2021), se exponen los resultados de una encuesta similar aplicada a los alumnos del Ciclo Introductorio Común (primer año).

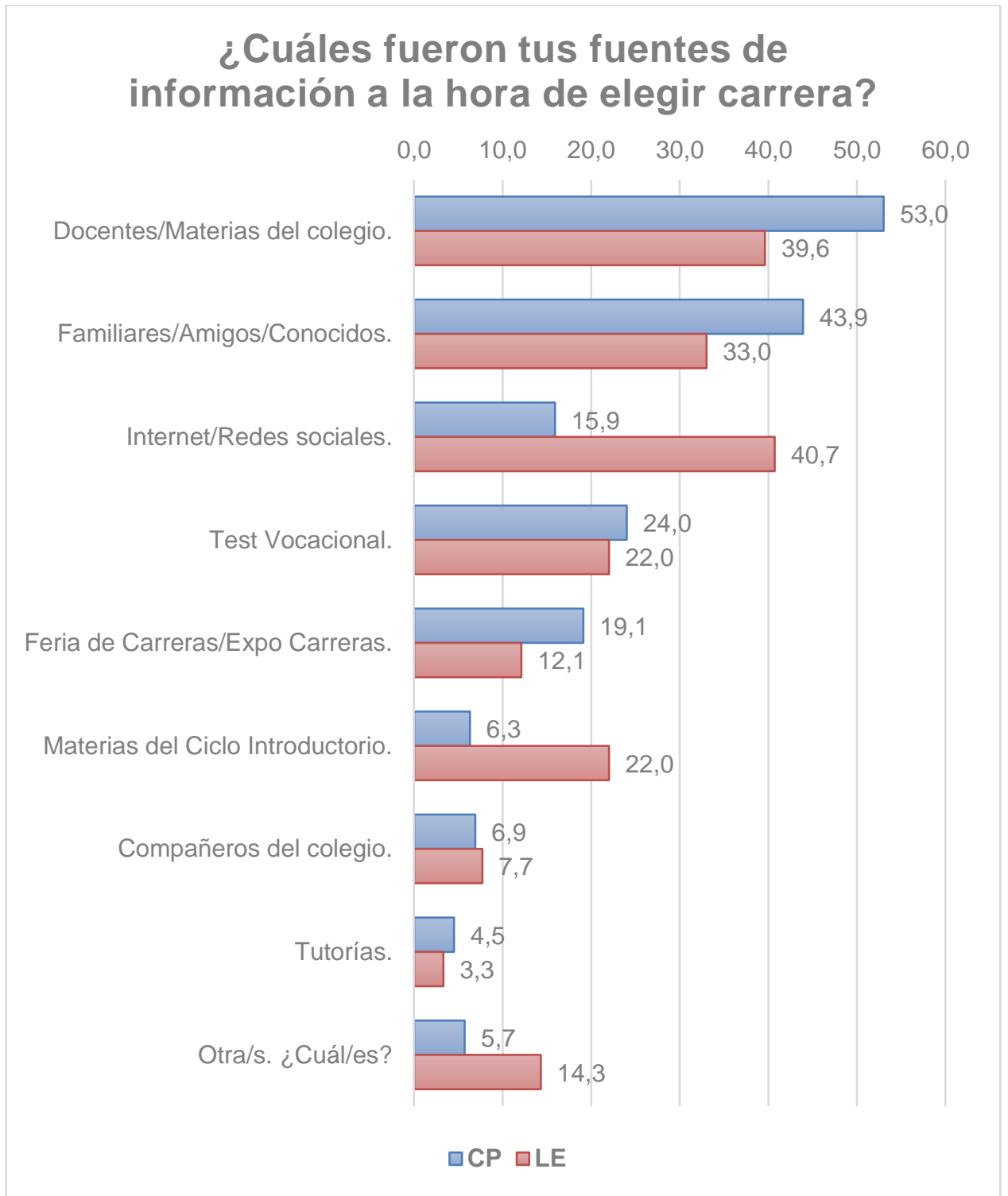

En cuanto a las fuentes de información al momento de la elección de la carrera y partiendo de la base de que es una pregunta de respuestas múltiples, los resultados son los siguientes:

- Para el caso de Contador Público, la fuente más seleccionada fue la proveniente de los docentes o materias del colegio secundario, con un $53 \%$, le sigue como segunda fuente los amigos, conocidos, y familiares con el 43,9\%; ya bastante por debajo 
están los test vocacionales (24\%), las ferias de Carreras $(19,1 \%)$ y las Redes Sociales e Internet con el 15,9\%. Ya con porcentajes más marginales, sin alcanzar el $10 \%$ ninguno de ellos, se encuentran como fuentes los compañeros del colegio secundario (6,9\%); las materias del ciclo Introductorio (6,3\%); las tutorías (4,5\%) y un $5,7 \%$ de otras fuentes.

- En lo que respecta a la Licenciatura en Economía, la fuente más seleccionada fue Internet y las Redes Sociales, con un 40,7\%, seguido de cerca por la proveniente de los docentes o materias del colegio secundario, con un $39,6 \%$, como tercera fuente aparecen los amigos, conocidos, y familiares con el 33\%; por debajo están los test vocacionales $(22 \%)$, las materias del ciclo Introductorio $(22 \%)$ y las ferias de Carreras $(12,1 \%)$. Con porcentajes menores se encuentran como fuentes los compañeros del colegio secundario $(7,7 \%)$ y las tutorías (3,3\%). Finalmente, con un $14,3 \%$ se encuentran otras fuentes.

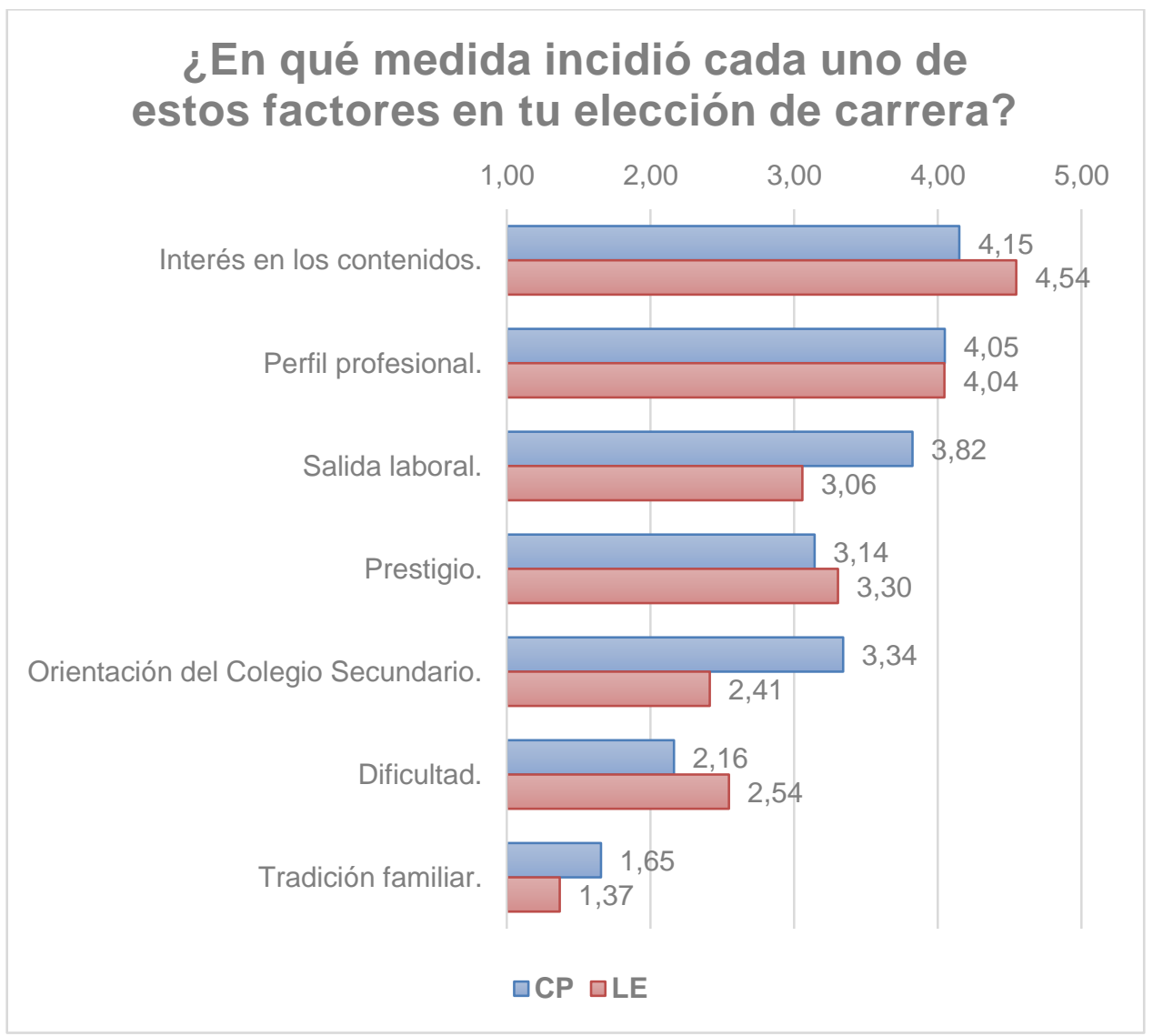

A la hora de evaluar de 1 a 5 (donde 1 es irrelevante y 5 es fundamental) qué tanto incidieron distintos factores a la hora de seleccionar la carrera, se encuentran los siguientes resultados:

- Para la carrera de Contador Público, el factor preponderante es el Interés en los contenidos con una media de 4,15 y una moda de 5. Le sigue como segundo factor mejor ponderado el Perfil profesional con una media de 4,05 y una moda de 5 . El 
tercer factor y muy vinculado con el precedente es la Salida Laboral con una media de 3,82 y como en los dos precedentes una moda de 5. El cuarto factor es la orientación del colegio secundario, con una media de 3,34, pero que llamativamente también tiene una moda de 5. Ya con menor peso se encuentra el Prestigio asociado al a profesión con una media de 3,14 y una moda de 3 . Finalmente, y ya con modas de 1 (es decir que con una alta frecuencia de encuestados que los consideran irrelevantes), se encuentran el grado de dificultad de la carrera (media de 2,16 ) y la Tradición Familiar (con una media de 1,65).

- Para el caso de la Licenciatura en Economía las valoraciones no difieren demasiado, el factor preponderante es el Interés en los contenidos con una media de 4,54 y una moda de 5. Le sigue como segundo factor mejor ponderado el Perfil profesional con una media de 4,04, también con una moda de 5 . El tercer factor es el Prestigio con una media de 3,30 y una moda de 4; al que le sigue la Salida Laboral con una media de 3,06 y una moda de 3 . El quinto factor es el grado de dificultad de la carrera (media de 2,54), le siguen la Orientación del Colegio Secundario $(2,41)$ y finalmente la Tradición Familiar (con una media de 1,37) para las tres opciones la moda es de 1, es decir Irrelevante.

\section{¿En qué momento decidiste seguilr Contador Público?}
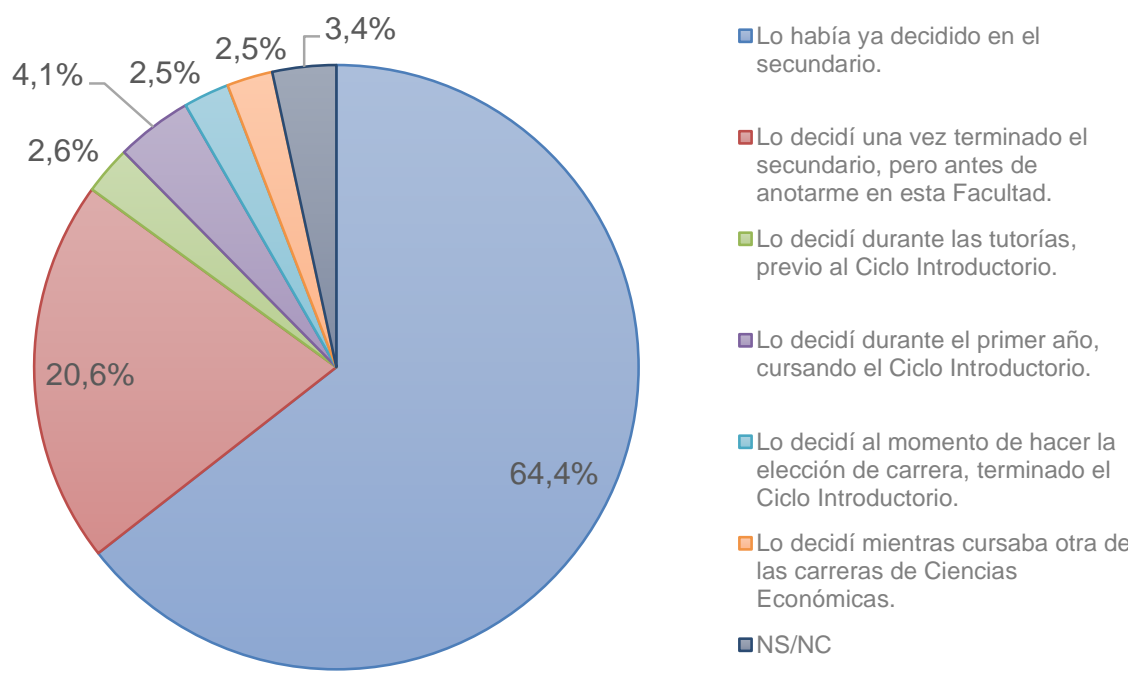

En lo que respecta al momento en que eligieron seguir la Carrera de Contador Público, la gran mayoría lo hizo antes de ingresar a la Facultad: el 64,4\% lo decidió durante la escuela secundaria y el $20,6 \%$ una vez terminado el secundario, pero antes de ingresar a la 
Facultad. Con menor frecuencia, algunos alumnos lo decidieron en los siguientes momentos: durante las tutorías previo al inicio del Ciclo Introductorio (2,6\%); durante el primer año en el Ciclo Introductorio (4,1\%); al momento de hacer la elección de Carrera (2,5\%); mientras cursaba otras Carreras de Ciencias Económicas (2,5\%).

\section{¿En qué momento decidiste seguilr Licenciatura en Economía?}
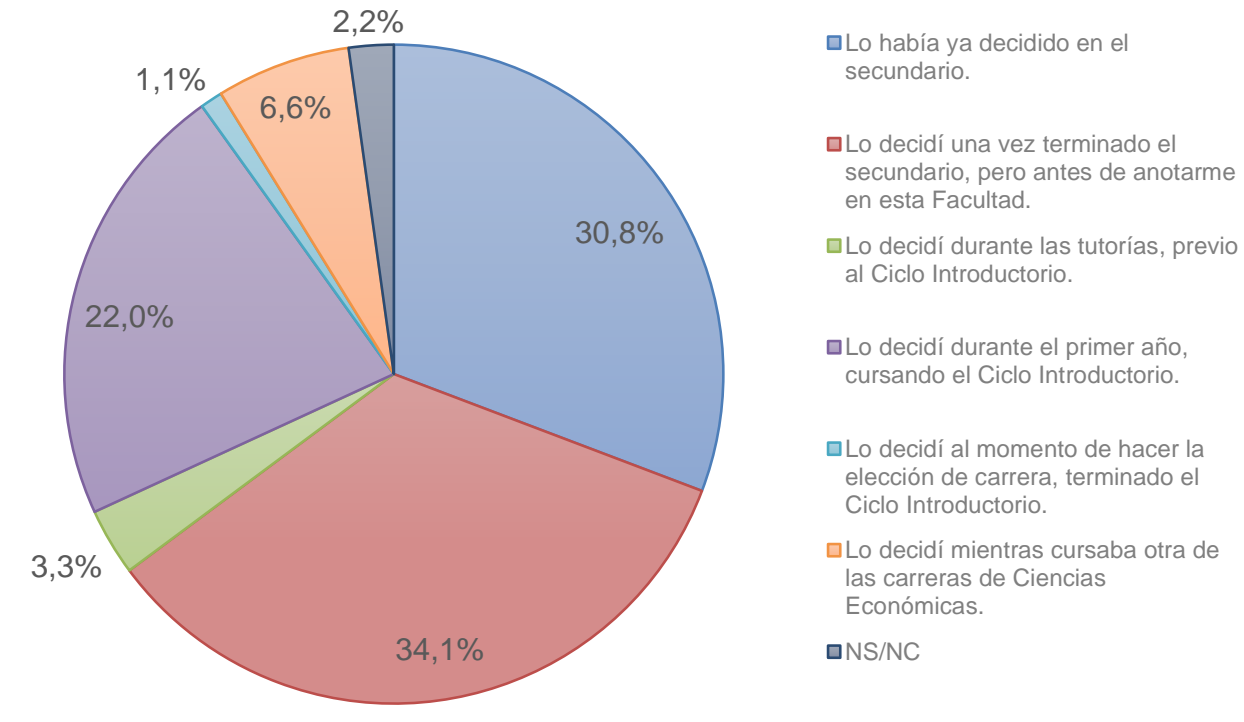

También en el caso de la Licenciatura en Economía, la mayoría decidió su carrera antes de ingresar a la Facultad: el 30,8\% lo decidió durante la escuela secundaria y el $34,1 \%$ una vez terminado el secundario, pero antes de ingresar a la Facultad. Un 22\% lo decidió durante el Ciclo Introductorio. Finalmente, y con menores frecuencias, algunos estudiantes lo decidieron durante las tutorías previo al inicio del Ciclo Introductorio (3,3\%), al momento de hacer la elección de Carrera $(1,1 \%)$ o mientras cursaba otras Carreras de Ciencias Económicas (6,6\%). 


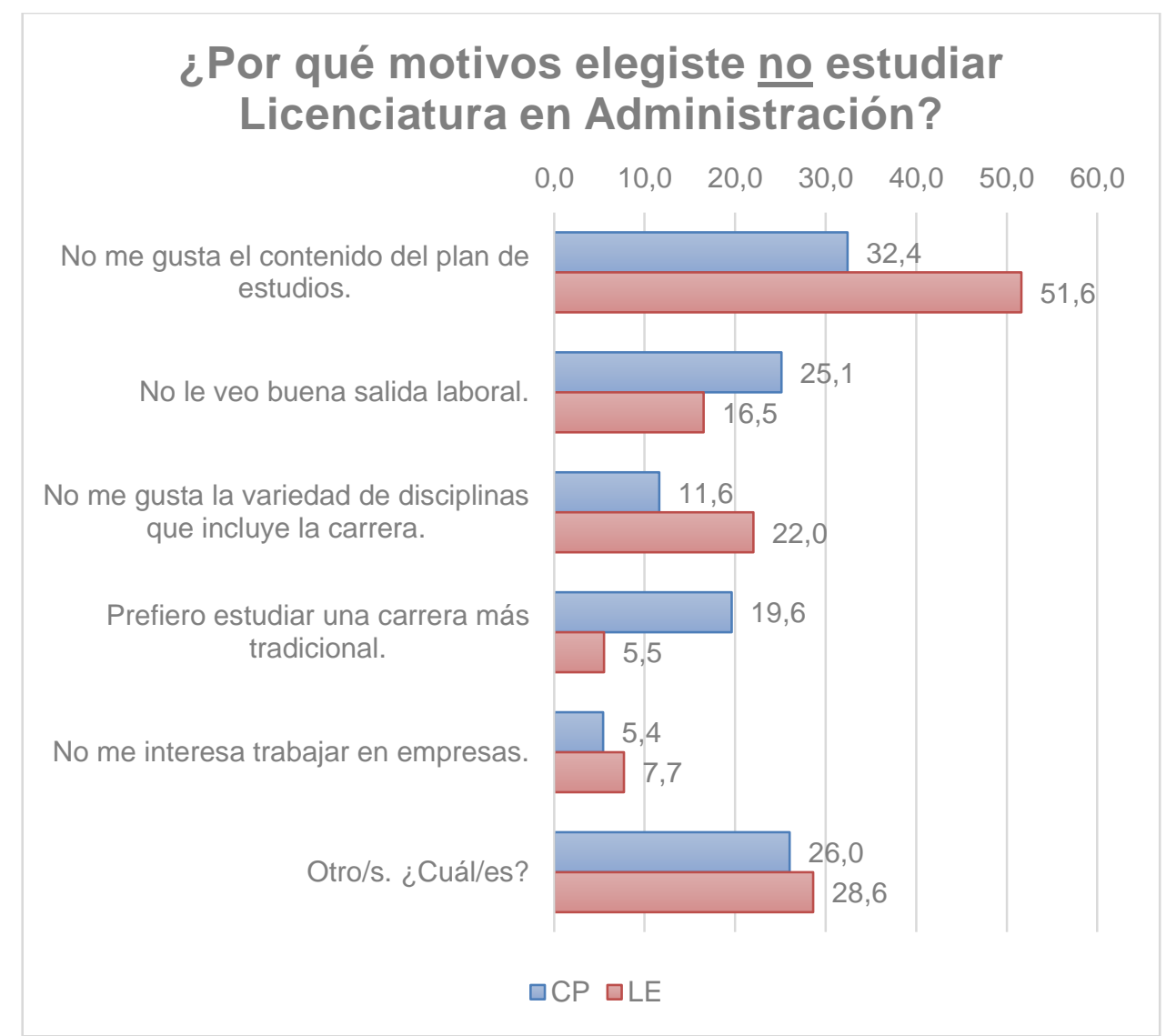

La pregunta siguiente se refirió a los motivos que llevaron al estudiante a no elegir la Licenciatura en Administración, pudiendo seleccionar todos los que apliquen.

Entre los estudiantes de Contador el motivo más seleccionado (32,4\%) es el contenido del plan de estudios, seguido por el hecho de no verle una buena salida laboral $(25,1 \%)$. Un $19,6 \%$ prefiere seguir una carrera más tradicional y a un $11,6 \%$ no le gusta la variedad de disciplinas que incluye la carrera; a un pequeño porcentaje no la considera atractiva por no interesarle el trabajo en empresas.

Un alto porcentaje (26\%) marco otros motivos, entre los que resaltan: la vocación y el gusto por la carrera de Contador Público; el desconocimiento de la Licenciatura en Administración; la falta de oferta de la Licenciatura en la mayor parte de las extensiones áulicas; la conveniencia en las equivalencias entre carreras de forma de estudiar primero Contador Público y luego hacer la Licenciatura en Administración. Algunos estudiantes en otros profundizaron alternativas ya presentadas, sobre todo la salida laboral, resaltando que la del Contador es más amplia, puede trabajar en forma independiente y puede firmar balances y en cuanto a los contenidos de las carreras, ya que consideran a la Licenciatura como demasiado generalista, sin un alto grado de profundidad, muy ambigua, poco técnica, muy teórica y con poca carga cuantitativa y ven como una desventaja la realización de la tesina o trabajo final. 
Algunas frases relevantes son las siguientes:

- "Consideré (quizás erróneamente) que la carrera de CP abarcaba la Lic en Administración; de todas maneras la idea es, una vez terminada la carrera $\mathrm{CP}$, hacer la Lic. en Adm.".

- "CPN puede hacer el trabajo de un LAE, pero en sentido inverso no".

- "Cuando hice Introducción a la Administración me di cuenta que no me gustaba entonces estudié Contador y en todo caso luego podía hacer las materias para Lic. en Adm.".

- "El plan de estudios tiene muchas similitudes con el de Contador, sin embargo siento que hay áreas como auditoria o impuesto que el administrador no puede ejercer mientras que el Contador sí puede tomar participación en áreas que corresponden a la Administración".

- "Falsa creencia en que la carrera apuntaba al marketing y la creatividad y no soy creativo".

- "He padecido las materias de Administración en nuestra carrera, no sé si están mal dadas, pero las hacen poco interesantes".

- "Me gusta la independencia que te da ser Contador Público, a diferencia de un administrador que generalmente trabaja en relación de dependencia".

- "Me parecía que la firma del Contador te habilitaba a cuestiones más amplias y, que en caso de querer y poder, podía complementar la carrera con algún estudio extra administrativo".

- "No noto una adecuada diferenciación entre la carrera de CP y la de LAE. Esto se demuestra al habiendo egresado de CP es mucho mas fácil/corto recibirse de LAE que el camino inverso".

- "No quería hacer la tesis".

- "Muchas veces un Contador puede hacer el trabajo de un administrador, un administrador el del Contador no."

- "Porque en el secundario miré las diferencias entre ambas y entendí en ese momento que el Lic. en Adm. era igual al CP pero solamente no podía firmar. A mitad del cursado confirmé que no era así".

- "Siento que la Carrera de CPN prácticamente incluye la Licenciatura en Administración de Empresas. CPN creo que es más abarcativa, y que como Contador uno tranquilamente también podría desarrollan cualquier tarea de un Licenciado en Administración de Empresas".

- "Un Contador tiene la misma salida laboral y además puede firmar balances". 
Para los estudiantes de Licenciatura en Economía, el motivo más seleccionado $(51,6 \%)$ es el contenido del plan de estudios, seguido por el hecho de no gustarle la variedad de disciplinas que incluye la carrera (22\%). El tercer factor más elegido es el de no verle una buena salida laboral (16,5\%). Un 5,5\% prefiere seguir una carrera más tradicional y un 7,7\% no elige porque no le interesa trabajar en empresas.

Un alto porcentaje $(28,6 \%)$ marcó otros motivos, entre los que sobresalen: la vocación por la carrera elegida; el desconocimiento de la Licenciatura en Administración; considerar la Licenciatura en Administración una carrera con menor contenido y menor exigencia; una falta de valoración por el trabajo del Licenciado en Administración. En cuanto a los contenidos de la carrera, consideran que la Licenciatura en Administración tiene un menor contenido y menor exigencia y prefieren la Licenciatura en Economía por ser menos técnica, con una mayor amplitud de criterios. Finalmente, al igual que en la carrera de Contador Público, algunos consideran una alternativa estudiar posteriormente la Licenciatura en Administración.

Algunos comentarios al respecto son los siguientes:

- "Desconocimiento, ya venía con la idea de estudiar Economía y nunca fue una opción".

- "Fue mi primera opción... Pero me dijeron que convenía Contador y después me dijeron que el Contador no viaja. Posteriormente me di cuenta q me gustaba la Economía".

- "La Lic. en Economía es menos técnica, buscaba la amplitud de criterios para ensamblar determinados escenarios".

- "La veía como una mixtura de otras carreras".

- "Me parecía bastante aburrido Introducción a la Administración".

- "Muchos egresados".

- "No le veo mucha dificultad, y me parece que alguien que no estudió pero que tiene buen trato con las personas puede ser mejor incluso que un/a Licenciado/a en Administración. No así con Economía".

- "Prefiero estudiar Licenciatura en Economía para entender las razones del crecimiento económico (de los países y las familias/personas) y, si se da la posibilidad hacer un aporte académico". 


\section{En tu opinión, ¿cuál es el ámbito de ejercicio propio del Licenciado en Administración?}

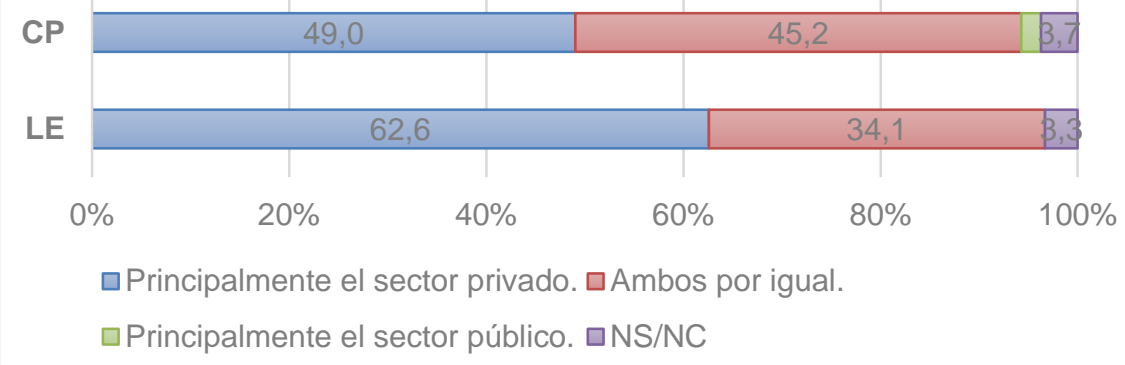

De un total de 734 estudiantes de la carrera de Contador Público encuestados, los resultados arrojan que $360(49,0 \%)$ consideran que el ámbito de ejercicio propio del Licenciado en Administración es principalmente el sector privado; mientras que 332 (45,2\%) encuestados opinan que es tanto el sector privado como el sector Público. Solo 15 (2\%) estudiantes de Contador Público visibiliza al ejercicio del Licenciado en Administración en el sector Público exclusivamente.

El porcentaje de respuestas entre "ambos por igual" y "principalmente sector privado" son similares, y entre ambos dejan muy poco margen para el ejercicio de la profesión exclusivamente en el sector Público.

Con respecto a las respuestas de los estudiantes de Licenciatura en Economía, sobre un total de 91 encuestados el 62.6\% (frecuencia de 57) considera que el ámbito de ejercicio del Licenciado en Administración es principalmente el sector privado y del $34.1 \%$ (31 encuestados) de ambos sectores por igual. La respuesta fue nula en torno a considerar exclusivamente el ámbito del sector Público.

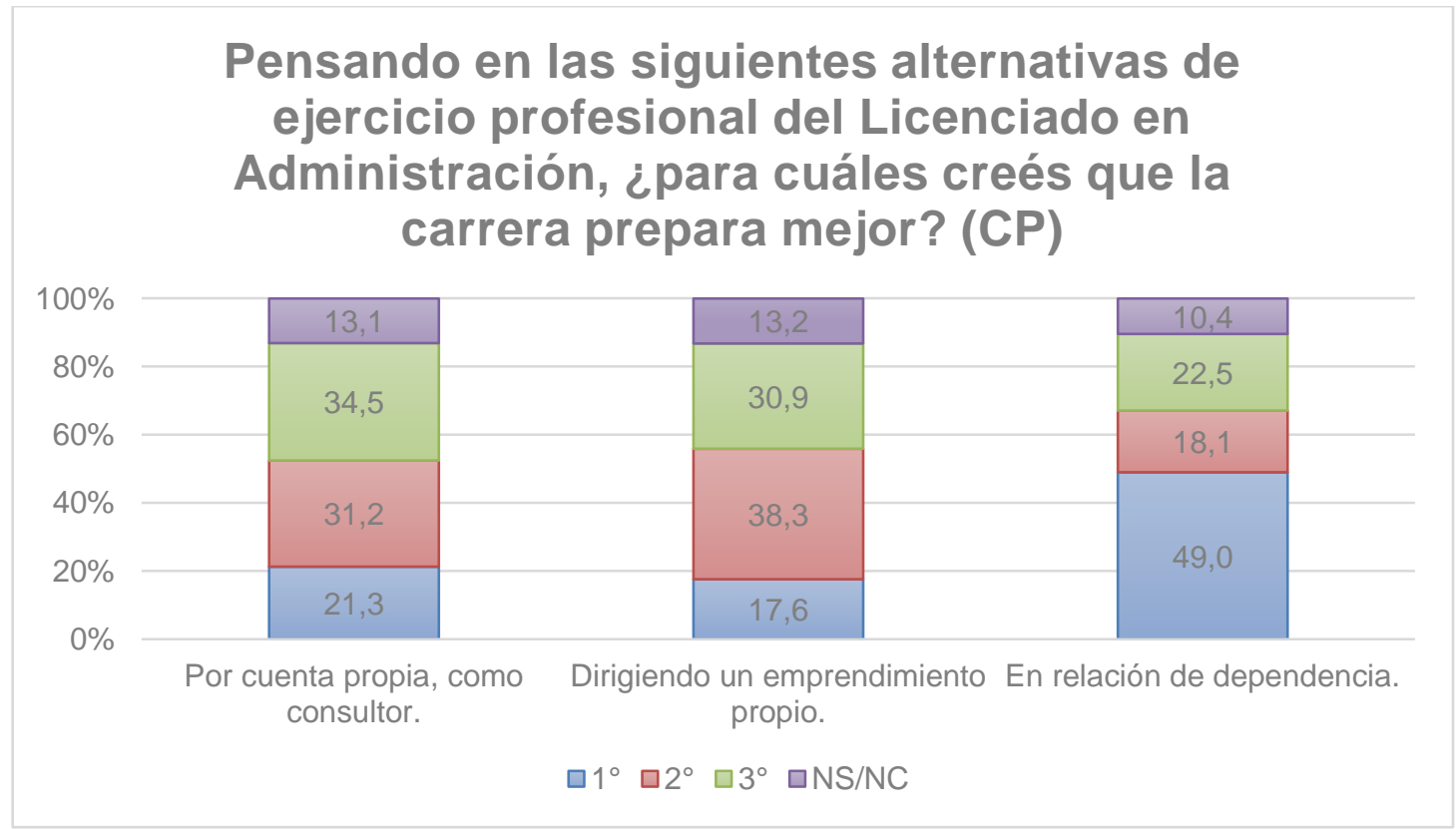


En lo que respecta a para qué alternativa la carrera de Licenciatura en Administración prepara mejor, los estudiantes de la carrera de Contador Público respondieron lo siguiente: el $49 \%$ considera que la carrera prepara mejor para desempeñarse en relación de dependencia (opción elegida por 360 encuestados); 17,6\% seleccionó "dirigiendo un emprendimiento propio" (129 encuestados) y 21,3\% (156 encuestados) seleccionaron "por cuenta propia como consultor".

En cuanto a cada una de las alternativas de ejercicio profesional para el Licenciado en Administración, las respuestas de los estudiantes de Contador Público fueron las siguientes: "Por cuenta propia como consultor", fue seleccionada por la mayoría como tercer opción con un $34,5 \%$; como segunda alternativa por el $31,2 \%$, y como primera por el $21,3 \%$ (un $13,1 \%$ no supo ubicarla en ninguna posición); "Dirigiendo un emprendimiento propio" fue seleccionada por la mayoría como segunda opción con un 38,3\%; como tercer alternativa por un $30,9 \%$ y como primera por el $17,6 \%$ (un $13,2 \%$ contesto NS/NC); finalmente "En relación de dependencia" fue tomada por la mayoría como la primer opción con un 49\%, como tercer opción por un $22,5 \%$ y como segunda opción por un $18,1 \%$ con un $10,4 \%$ que no la ubican en ninguna posición.

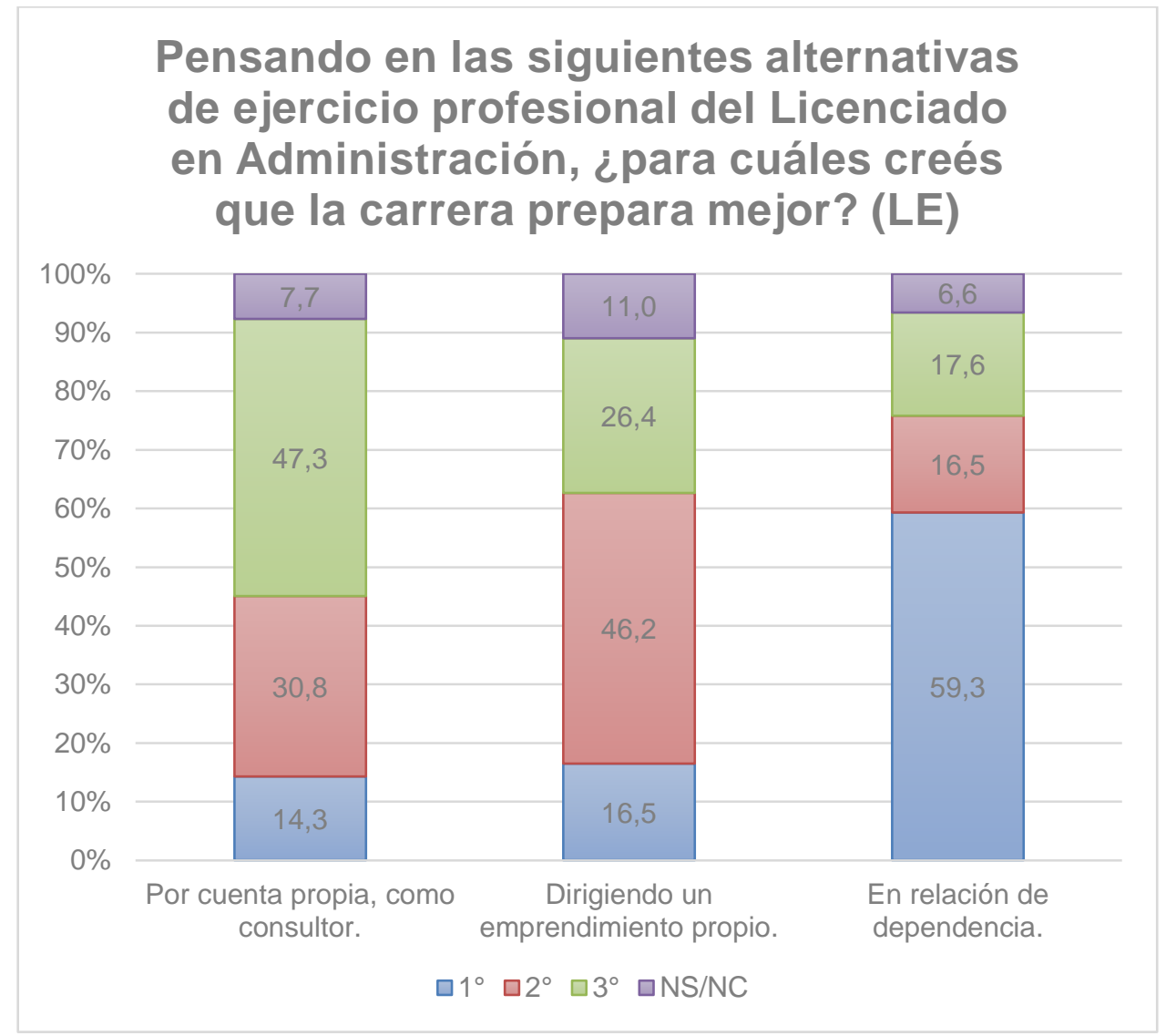

En lo que respecta a los estudiantes de Licenciatura en Economía, el 59,3\% de los encuestados considera que la carrera Licenciatura en Administración prepara mejor para desempeñarse "en relación de dependencia", un 16.5\% considera que prepara mejor para 
"dirigir un emprendimiento propio" mientras que un 14,3\% ubica en primer lugar a "por cuenta propia como consultor".

En lo que respecta a cada una de las alternativas de ejercicio profesional para el Licenciado en Administración, las respuestas de los estudiantes de Licenciatura en Economía fueron las siguientes: "Por cuenta propia como consultor", fue seleccionada por la mayoría como tercer opción con un 47,3\%; como segunda alternativa por el 30,8\%, y como primera por el $14,3 \%$ (un 7,7\% no la ubicó en ninguna posición); "Dirigiendo un emprendimiento propio" fue seleccionada por la mayoría como segunda opción con un $46,2 \%$; como tercer alternativa por un $26,4 \%$ y como primera por el $16,5 \%$ (un $11 \%$ contesto NS/NC); finalmente "En relación de dependencia" fue tomada por la mayoría como la primer opción con un 59,3\%, como tercer opción por un 17,6\% y como segunda opción por un $16,5 \%$ con un $6,6 \%$ que no la ubica en ninguna posición.

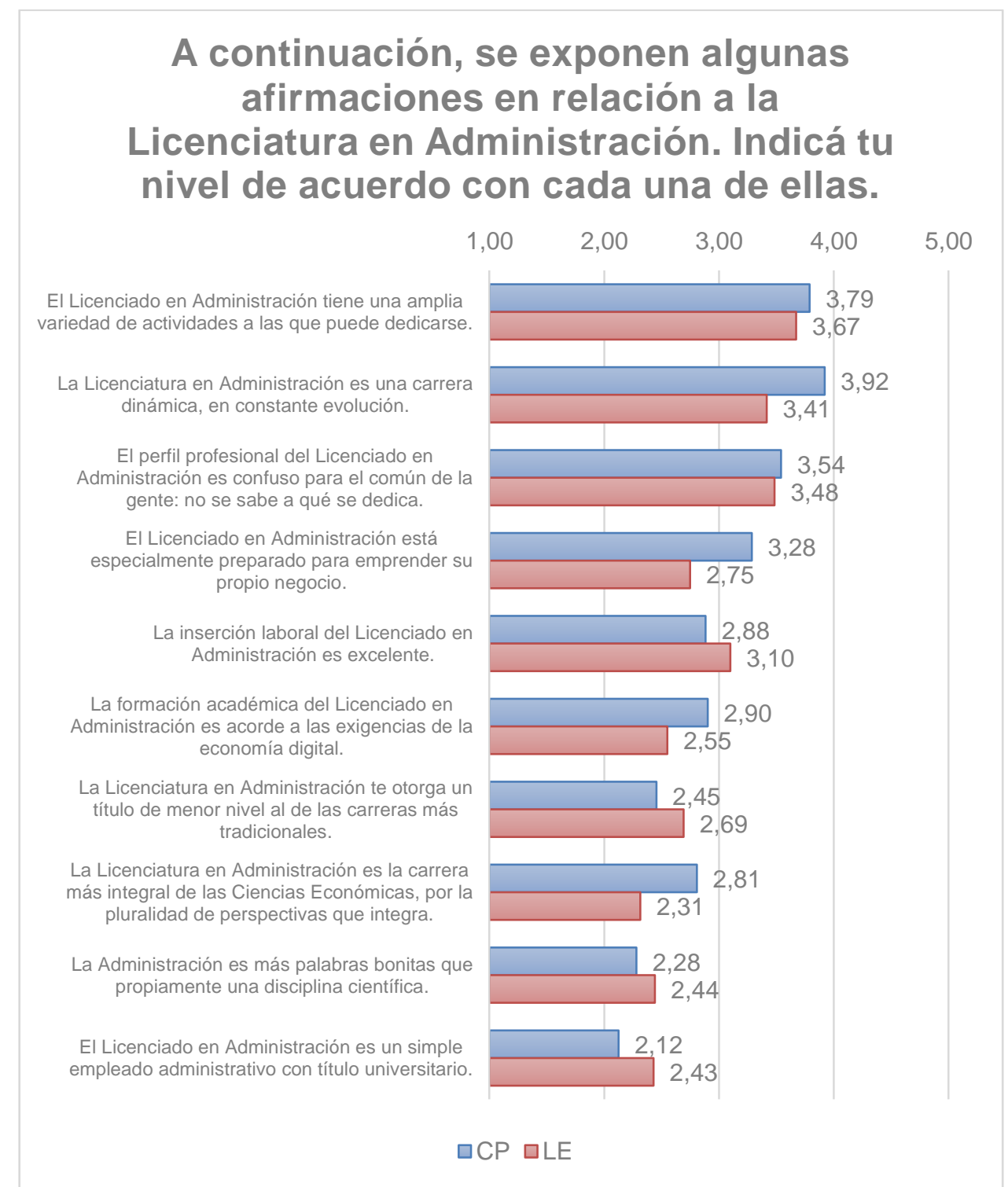

La pregunta siguiente se orienta a relevar la imagen de la Licenciatura en Administración por medio del nivel de acuerdo con una serie de afirmaciones sobre la carrera. 
En cuanto a los alumnos de Contador Público, las afirmaciones con mayor nivel de acuerdo promedio son: "La Licenciatura en Administración es una carrera dinámica, en constante evolución" con una media de 3,92, "El Licenciado en Administración tiene una amplia variedad de actividades a las que puede dedicarse" con una media de 3,79 , le sigue "El perfil profesional del Licenciado en Administración es confuso para el común de la gente: no sabe a qué se dedica con una media de 3,54. Todas ellas con una mediana de 4 y una moda de 5.

Aquellas afirmaciones con una mediana y moda de 3 , se expresan a continuación ordenadas según el valor de la media: "El Licenciado en Administración está especialmente preparado para emprender su propio negocio" $(3,28)$; "La formación académica del Licenciado en Administración es acorde a las exigencia de la Economía digital" (2,90); "La inserción laboral del Licenciado en Administración es excelente" $(2,88)$ y "La Licenciatura en Administración es la carrera más integral de las Ciencias Económicas por la pluralidad de perspectivas que integra" $(2,81)$.

Aquellas afirmaciones que tienen el menor nivel de acuerdo, que cuentan con una mediana de 2 y moda de 1, se expresan a continuación ordenadas según el valor de la media: "La Licenciatura en Administración te otorga un título de menor nivel al de las carreras más tradicionales" (2,45); "La Administración es más palabras bonitas que una disciplina científica" (2,28); "El Licenciado en Administración es un simple empleado administrativo con título universitario" $(2,12)$.

Para los estudiantes de Licenciatura en Economía la afirmación "El Licenciado en Administración tiene una amplia variedad de actividades a la que puede dedicarse" es en promedio la que tiene mayor nivel de acuerdo entre los encuestados con una media de 3,67 y una mediana de 4 , y el mismo valor para la moda.

Las afirmaciones que arrojaron una mediana y una moda de 3 son las siguientes, ordenadas según la media: "El perfil profesional del Licenciado en Administración es confuso para el común de la gente: no sabe a qué se dedica" $(3,48)$; "La Licenciatura en Administración es una carrera dinámica, en constante evolución" (3.41); La inserción laboral del Licenciado en Administración es excelente" $(3,10)$; "El Licenciado en Administración está especialmente preparado para emprender su propio negocio" (2,75); "La formación académica del Licenciado en Administración es acorde a las exigencias de la Economía digital" $(2,69)$.

Por último, las afirmaciones que tienen una mediana de 3 y una moda de 1 son las siguientes en orden de su media: "La Licenciatura en Administración te otorga un título de menor nivel al de las carreras tradicionales" $(2,69)$; "La Administración es más palabras bonitas que propiamente una disciplina científica" $(2,44)$; "El Licenciado en Administración es un simple empleado administrativo con título universitario" $(2,43)$; "La Licenciatura en 
Administración es la carrera más integral de las Ciencias Económicas por la pluralidad de perspectivas que integra" $(2,31)$.

Para cerrar, la última pregunta del cuestionario se propone medir hasta dónde tienen los sujetos claro cuáles son las competencias específicas de un Licenciado en Administración. A tal fin, se les ofrece un listado de 15 competencias específicas, de las cuales siete fueron tomadas del plan de estudios de la Licenciatura en Administración (Resolución № 28982C.D.), cuatro del plan de estudios de Contador Público (Resolución № 27817-C.D.) y otras cuatro del plan de carrera de la Licenciatura en Economía (Resolución № 28981-C.D.).

Para cada una de estas competencias específicas, se solicitó a los encuestados que indique si se trata de una competencia propia del Licenciado en Administración, si le compete, pero no exclusivamente, o si simplemente no le compete. Si bien a continuación se exponen las competencias ordenadas según de qué plan de estudios fueron extraídas (Primera parte: LA; Segunda parte: CP; Tercera parte; LE), mostrándose incluso en gráficos separados, a los encuestados se les presentaron en forma aleatoria.

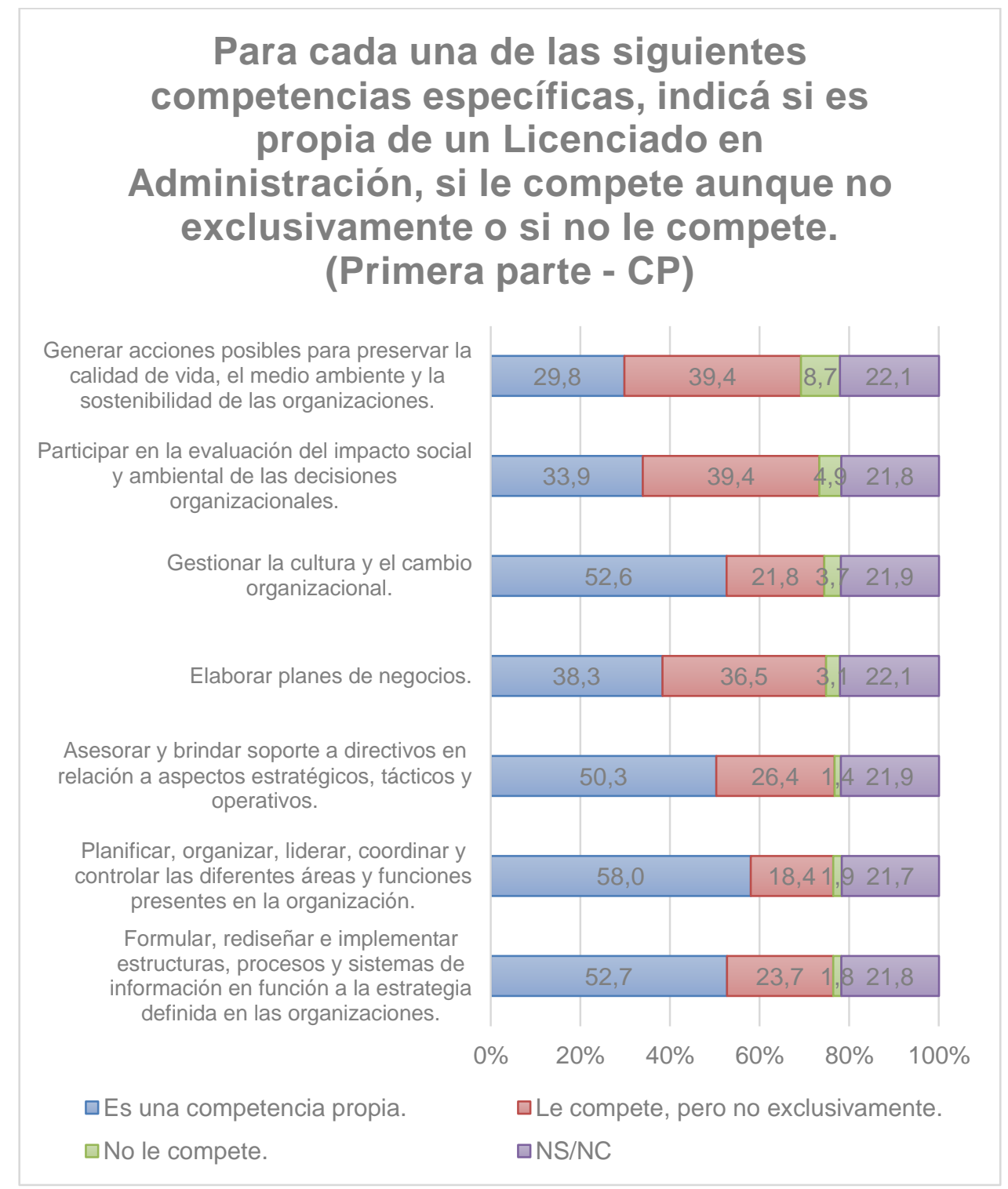


De esta primera parte del listado de competencias, aquellas competencias que el estudiante de Contador Público consideró como específicas de la Licenciatura en Administración y que tienen una respuesta con un porcentaje mayor al 50,0\% son las siguientes: "Planificar, organizar, liderar, coordinar y controlar las diferentes áreas y funciones presentes en la organización" frecuencia de 426 (58,0\%); seguido por una frecuencia de $387(52,7 \%)$ la competencia "Formular, rediseñar e implementar estructuras, procesos y sistemas de información en función a la estrategia definida en las organizaciones" y luego la competencia "Gestionar la cultura y el cambio organizacional" con una frecuencia de 386 $(52,6 \%)$ y "Asesorar y brindar soporte a directivos en relación a aspectos estratégicos, tácticos y operativos" con 369 (50,3\%).

Con respecto a "Le compete, pero no exclusivamente" aquellas competencias que tienen un mayor porcentaje de respuesta son: "Generar acciones posibles para preservar la calidad de vida, el medio ambiente y la sostenibilidad de las organizaciones"; "Participar en la evaluación del impacto social y ambiental de las decisiones organizacionales" ambas con una frecuencia de 289 (39,4\%). "Elaborar planes de negocio" es una competencia que tuvo un porcentaje similar en la respuesta competencia propia (38.3\%) y no exclusiva (36.5\%).

\section{Para cada una de las siguientes competencias} específicas, indicá si es propia de un Licenciado en Administración, si le compete aunque no exclusivamente o si no le compete. (Primera parte - LE)

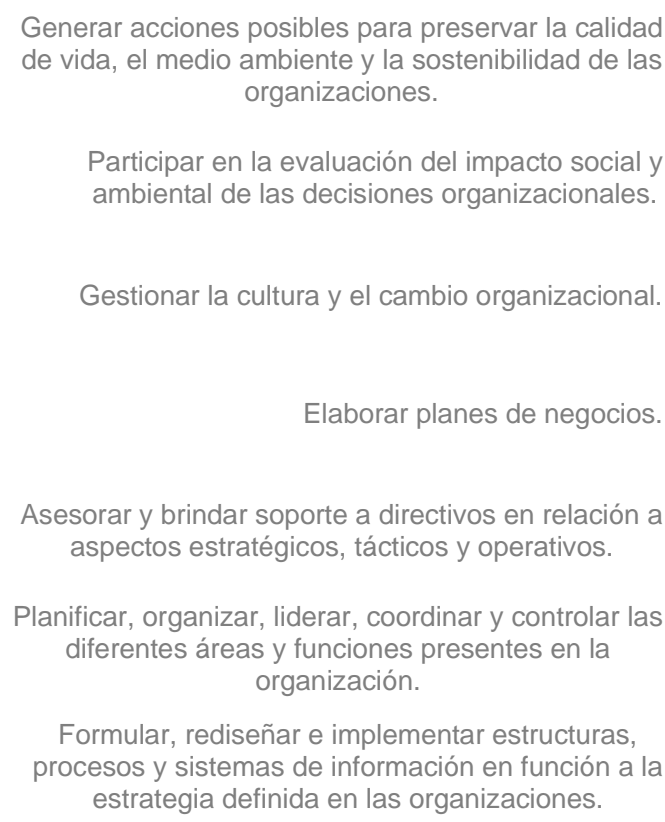

Formular, rediseñar e implementar estructuras, procesos y sistemas de información en función a la estrategia definida en las organizaciones.

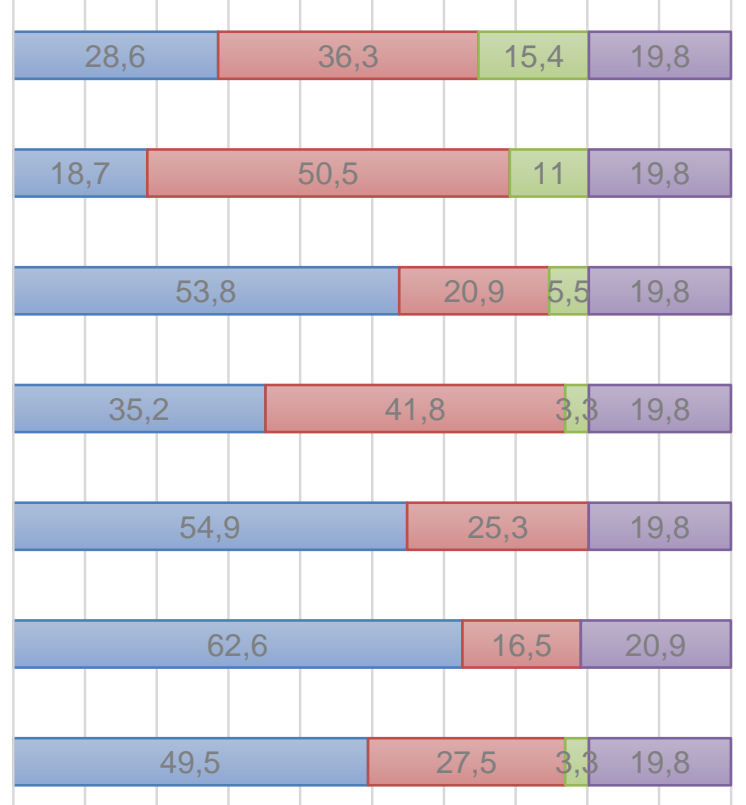

0\% 10\% 20\% 30\% 40\% 50\% 60\% 70\% 80\% 90\% $100 \%$

$\square$ Es una competencia propia. $\square$ Le compete, pero no exclusivamente. $\square$ No le compete. $\square$ NS/NC 
Para los encuestados de la carrera Licenciatura en Economía, en lo que respecta a aquellas competencias específicas propias del Licenciado en Administración de esta primera parte, aparecen las siguientes: "Planificar, organizar, liderar, coordinar y controlar las diferentes áreas y funciones presentes en la organización" con 57 respuestas (62,6\%); seguida por "Asesorar y brindar soporte a directivos en relación a aspectos estratégicos, tácticos y operativos" con una frecuencia de 50 (54,9\%); con el 53,8\% (49 respuestas) sigue la competencia: "Gestionar la cultura y el cambio organizacional" y "Formular, rediseñar, e implementar estructuras, procesos y sistemas de información en función a la estrategia definida en las organizaciones" con una frecuencia de 45 (49,5\%).

Aquellas competencias que en general consideran les corresponde pero no exclusivamente se pueden observar los siguientes resultados: "Participar en la evaluación social y ambiental de las decisiones organizacionales" (50,5\%) frecuencia 46; "Elaborar planes de negocios" $(41,8 \%)$ frecuencia 38 ; "Generar las acciones posibles para preservar la calidad de vida, el medio ambiente y la sostenibilidad de las organizaciones" con una frecuencia de 33 $(36,3 \%)$. Esta misma competencia fue aquella que, dentro de las respuestas "no le compete", arrojó el mayor porcentaje $(15,4 \%)$.

\section{Para cada una de las siguientes competencias específicas, indicá si es propia de un Licenciado en Administración, si le compete aunque no exclusivamente o si no le compete. (Segunda parte - CP)}

Realizar los procesos de sindicatura en sociedades, concursos y quiebras.

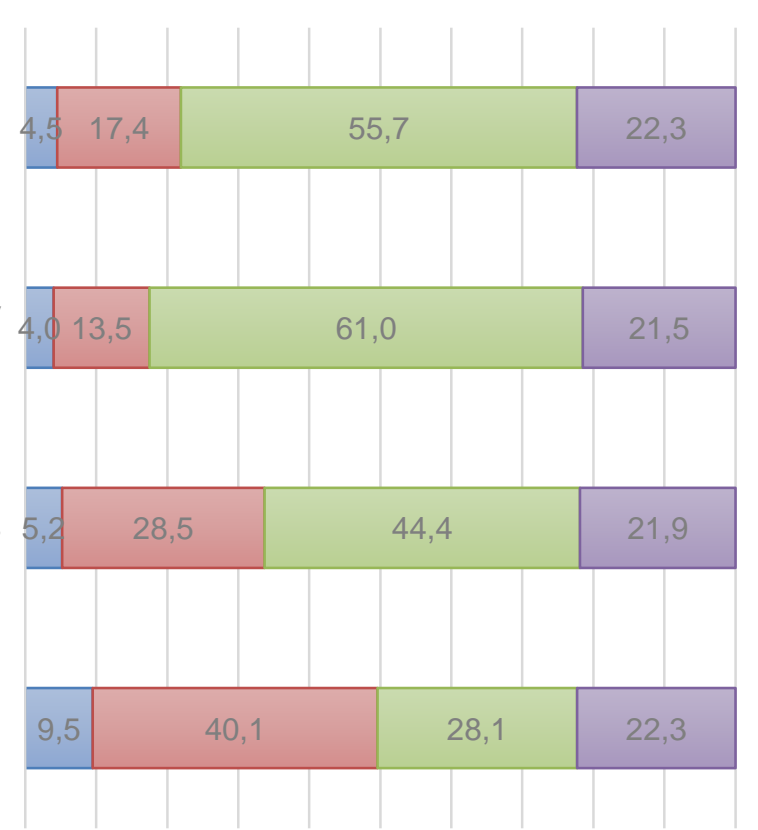

Dirigir y realizar procedimientos de auditoría, y dictaminar en materia contable e impositiva.

Registrar, medir y exponer la información contable, histórica y proyectada, para todo tipo de organizaciones y unidades económicas.

Diseñar, dirigir e implementar sistemas de información contable en todos los segmentos de la contabilidad y costos.

0\% 10\% 20\% 30\% 40\% 50\% 60\% 70\% 80\% 90\% 100\%

$\square$ Es una competencia propia. $\square$ Le compete, pero no exclusivamente. $\square$ No le compete. $\square$ NS/NC 
Respecto a la segunda parte, que son aquellas competencias extraídas del plan de Contador Público, aquellas competencias que los estudiantes de dicha carrera consideran que no les compete al Licenciado en Administración son: "Dirigir y realizar procedimientos de auditoría y dictaminar en materia contable e impositiva" con una frecuencia de 448 $(61,0 \%)$; seguido de "Realizar procesos de sindicaturas en sociedades, concursos y quiebras" con una frecuencia de 409 (55,7\%), "Registrar, medir y exponer información contable histórica y proyectada, para todo tipo de organizaciones y unidades económicas" con una cantidad de respuestas de 326 (44,4\%).

Con respecto a "Diseñar, dirigir e implementar sistemas de información contable en todos los segmentos de la contabilidad y costos" la mayoría de los encuestados respondió, a diferencia de las anteriores, que es una competencia más no exclusiva del Licenciado en Administración con una cantidad de respuestas de 294 (40,1\%) frente a un 28,1\% (206 respuestas) que considera no le compete.

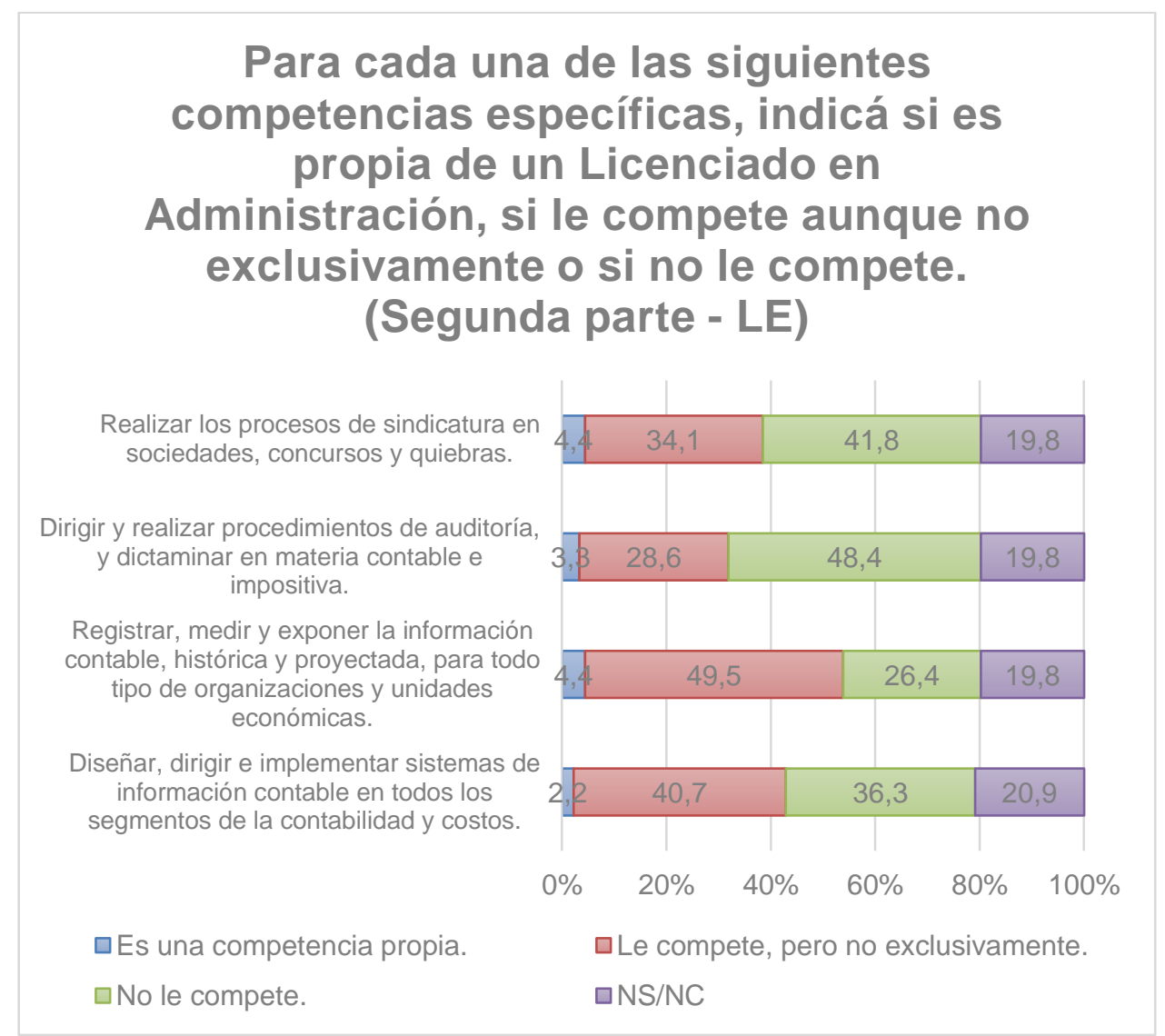

En el caso de los estudiantes de la Licenciatura en Economía, consideran que no le compete al Licenciado en Administración "Dirigir y realizar procedimientos de auditoría y dictaminar en materia contable e impositiva" (48,4\%, 44 respuestas); "Realizar los procesos de sindicaturas en sociedades, concursos y quiebras" (41,8\%, 38 respuestas).

Consideran en su mayoría que les compete pero no exclusivamente: "Registrar, medir y exponer información contable histórica y proyectada para todo tipo de organizaciones y 
unidades económicas" (49,5\%, 45 respuestas); "Diseñar, dirigir e implementar sistemas de información contable en todos los segmentos de la contabilidad y costos" $(40,7 \%, 37$ respuestas).

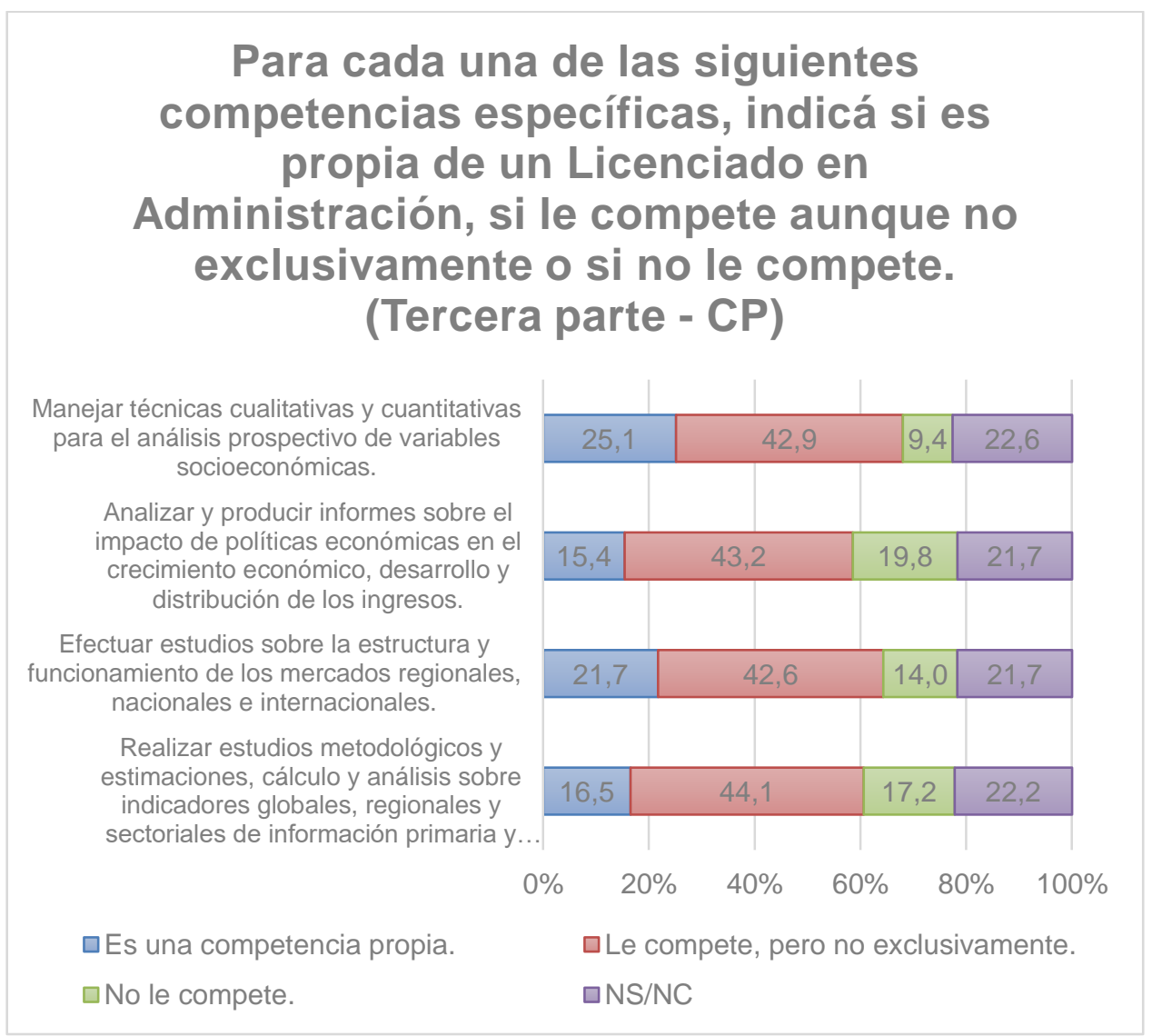

Para todas las competencias específicas citadas en la tercera parte de la pregunta, que son las competencias extraídas del plan de Licenciatura en Economía, los estudiantes de Contador Público consideraron que le competen al Licenciado en Administración, más no exclusivamente. En orden según el porcentaje de respuesta se detalla a continuación: "Realizar estudios metodológicos y estimaciones, cálculo y análisis sobre indicadores globales, regionales y sectoriales de la información primaria y secundaria" con una frecuencia de 324 (44,1\%), seguido por "Analizar y producir informes sobre el impacto de políticas económicas en el crecimiento económico, desarrollo y distribución de los ingresos" con una cantidad de respuestas $317(43,2 \%)$; luego "Manejar técnicas cuantitativas y cualitativas para el análisis prospectivo de variables socioeconómicas" con una frecuencia de 315 (42,9\%) y, por último, "Efectuar estudios sobre la estructura y funcionamiento de los mercados regionales, nacionales e internacionales" con una frecuencia de $313(42,6 \%)$.

Es interesante mencionar que en la primera y tercera competencia mencionada anteriormente, el porcentaje de respuesta entendiéndola como competencia específica propia de la Licenciatura en Administración supera a la respuesta de "no le compete". El 
caso inverso sucede con la segunda y cuarta competencia. Siempre teniendo en cuenta que se está analizando el punto de vista y percepción del estudiante de Contador Público.

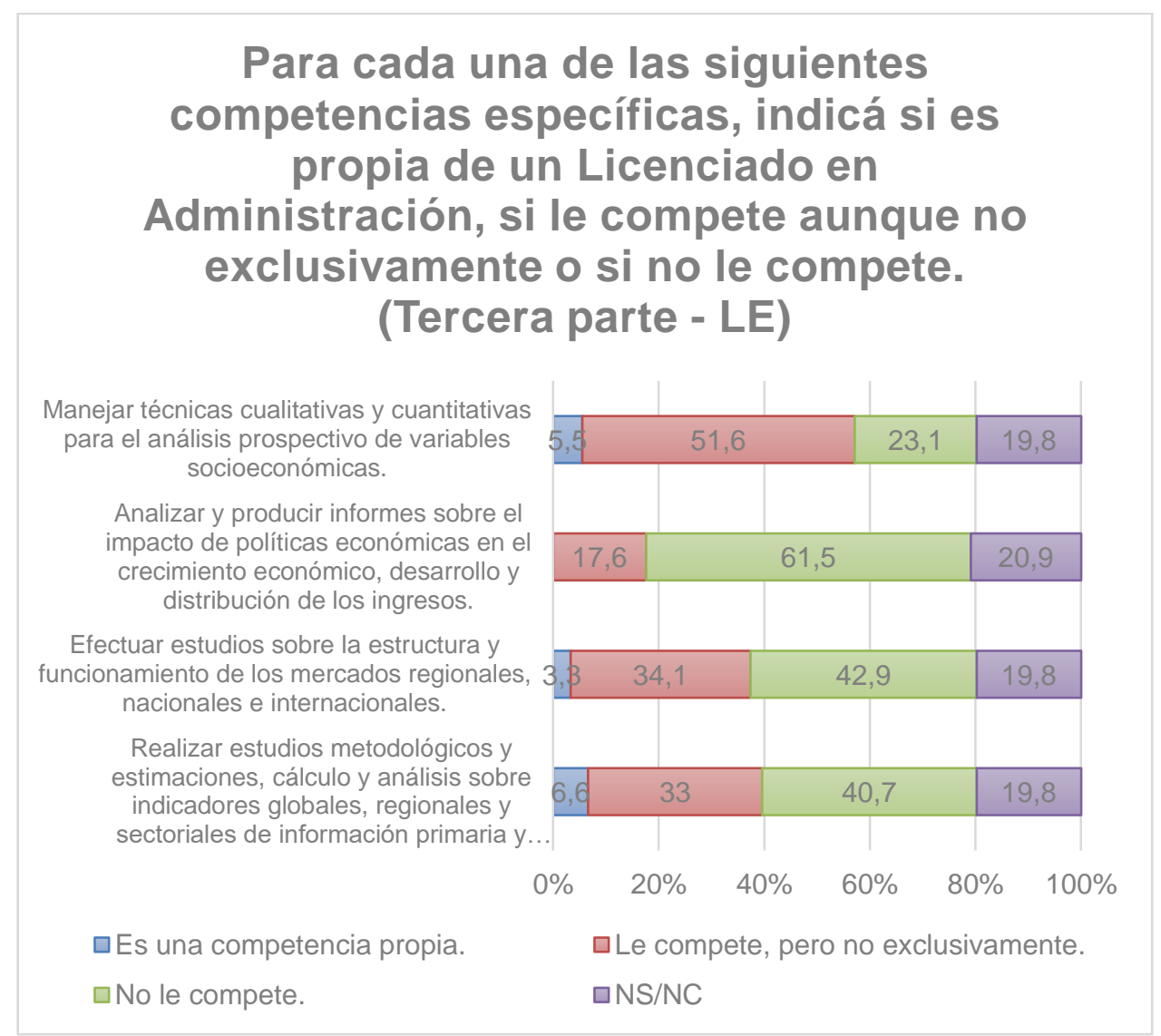

En el caso de los estudiantes de Licenciatura en Economía consideran en su mayoría que las siguientes competencias específicas no le competen a los Licenciados en Administración: "Analizar y producir informes sobre el impacto de políticas económicas en el crecimiento económico, desarrollo y distribución de ingresos" con 56 respuestas $(61,5 \%)$; "Efectuar estudios sobre la estructura y funcionamiento de los mercados regionales, nacionales e internacionales" con 39 respuestas (42,9\%); "Realizar estudios metodológicos y estimaciones, cálculo y análisis sobre indicadores globales, regionales y sectoriales de información primaria y secundaria" con una frecuencia de 37 (40,7\%).

Con respecto a la competencia específica "Manejar técnicas cuantitativas y cualitativas para el análisis prospectivo de variables socioeconómicas" entienden le compete al Licenciado en Administración pero no exclusivamente.

\section{CONCLUSIONES}

El presente artículo se basó en un relevamiento realizado a una muestra representativa de estudiantes de las carreras de Contador Público (734 encuestas) y de la Licenciatura en Economía (91 encuestas). En la muestra hubo mayoría de género femenino para Contador Público $(70,7 \%)$ y mayoría de género masculino para Licenciatura en Economía (62,6\%), la 
edad promedio de los participantes fue de 26,09 años para Contador y 26,16 para Economía, y en ambos casos la mayor parte de los encuestados se encuentra en el segundo ciclo de la carrera (en cuarto y quinto año).

La primera parte de la encuesta estuvo dirigida a conocer el proceso de selección de carrera y a los motivos para no seguir la Licenciatura en Administración. De los resultados se desprenden las siguientes conclusiones:

- En cuanto a la principal fuente de información por la que los estudiantes conocieron y se interesaron en la carrera hay diferencia entre Contador y Economía; en el primer caso fueron los docentes o las materias del colegio secundario mientras que para la Licenciatura fueron Internet y las Redes Sociales, siendo el colegio secundario la segunda fuente en este caso. En ambos casos tuvieron peso las referencias de amigos, conocidos y familiares (segunda fuente para Contador y tercera para Economía).

- A la hora de seleccionar la carrera también hubo fuertes similitudes entre ambas carreras; el factor preponderante es el Interés en los contenidos (en ambos casos con una moda de 5) al que le sigue el perfil profesional (con moda de 5). En el caso de Contador también tiene una moda de 5 el tercer factor que es la salida laboral, no tan relevante para los futuros Licenciados en Economía (moda de 3). Entre los factores menos importantes se destacan para las dos carreras el grado de dificultad y la tradición familiar, ambos con moda de 1 (irrelevantes). Llama la atención que para los estudiantes de Economía también ha sido irrelevante la orientación del colegio secundario.

- $\quad$ En cuanto al momento de la elección la mayoría lo hizo antes de ingresar a la Facultad en las dos carreras.

- $\quad$ En lo que respecta a los motivos que los llevaron a no elegir la Licenciatura en Administración sobresale el contenido del plan de estudios tanto para los estudiantes de Contador como los de la Licenciatura en Economía. Para los estudiantes de Contador un segundo factor relevante es la salida laboral, ya que consideran que la carrera de Contador Público tiene una mayor salida laboral, más amplia, con posibilidades de trabajo por cuenta propia y la posibilidad de "firmar balances". Entre los Otros motivos que podían agregar se hizo hincapié en la vocación, el desconocimiento de la carrera y la falta de oferta de la Licenciatura en las Extensiones Áulicas. En el caso de los estudiantes de la Licenciatura en Economía la segunda alternativa más seleccionada fue la variedad de disciplinas que incluye la carrera dejando como tercer factor la salida laboral. Dentro de los otros motivos, al igual que para los alumnos de Contador sobresalen la vocación por la carrera elegida y el desconocimiento de la 
Licenciatura en Administración; y aparecen como otros factores considerar la Lic en Administración una carrera con menor contenido y menor exigencia, con una desvalorización del trabajo del Licenciado en Administración.

Algo interesante es que en las dos carreras un buen número de alumnos manifestaron la intención de comenzar posteriormente la Licenciatura en Administración.

La segunda parte de la encuesta está orientada a la percepción de los estudiantes de Contador Público y Licenciatura en Economía en cuanto al desempeño en el ámbito laboral de los Licenciados en Administración. En este punto las principales conclusiones son:

- La mayoría de los estudiantes de Contador Público y los alumnos de la Licenciatura en Economía coinciden que el ámbito de ejercicio propio del Licenciado en Administración es el sector privado, ya sea exclusivamente o combinado con el sector Público. Esta identificación de la Licenciatura en Administración con en el sector privado tiene una mayor preponderancia en el caso de los estudiantes de la Licenciatura en Economía.

- $\quad$ En lo que respecta al ejercicio profesional, los estudiantes encuestados tanto de Licenciatura en Economía como de Contador Público coinciden que la Licenciatura en Administración prepara mejor para desempeñarse en relación de dependencia en primer lugar, luego para dirigir un emprendimiento propio y por último por cuenta propia como consultor. En el caso de las respuestas de Licenciatura en Economía el porcentaje que ubicó a relación de dependencia en primer lugar fue mayor que el correspondiente a Contador Público.

- En cuanto a las afirmaciones en relación a la Licenciatura en Administración que generaron mayor acuerdo se encuentran: "El Licenciado en Administración tiene una amplia variedad de actividades a la que puede dedicarse"; "La Licenciatura en Administración es una carrera dinámica en constante evolución"; "El profesional que forma está preparado para emprender su propio negocio" y "El perfil profesional es confuso para el común de la gente". Los encuestados de la carrera Licenciatura en Economía tuvieron un mayor nivel de acuerdo, en comparación con las respuestas de Contador Público, en la afirmación "La salida laboral del Licenciado en Administración", mientras que, por otro lado, los encuestados de la carrera de Contador en su mayoría demuestran tener un mayor nivel de acuerdo en percibir a la Licenciatura en Administración como la carrera más integral de las Ciencias Económicas por la pluralidad de perspectivas que integra.

- $\quad$ Finalmente, en lo que respecta a las competencias específicas si bien en general hay una identificación de las competencias propias de cada carrera, se detecta 
que para los estudiantes es más claro diferenciar la carrera que está cursando de las otras dos, que las otras dos entre sí.

Los resultados en las respuestas de los encuestados de ambas carreras coinciden en las siguientes competencias específicas a las cuales le otorgaron el mayor porcentaje: "Planificar, organizar, liderar, coordinar y controlar las diferentes áreas y funciones presentes en la organización”, gestionar la cultura y cambio organizacional, asesoramiento a directivos y en alinear procesos, información y estructura a la estrategia organizacional. Coinciden en que las gestiones de sustentabilidad y aquellas referidas a la evaluación social y ambiental no les son exclusivas.

Sin embargo, en la competencia "elaborar planes de negocios", los encuestados de la Licenciatura en Economía arrojaron un porcentaje mayor en entenderla como no exclusiva y las respuestas de Contador Público se corresponden a un porcentaje similar entre propias y no exclusivas.

Los resultados arrojados por ambas carreras coinciden en que no están dentro de las competencias del Licenciado en Administración aquellas relacionadas con procesos de sindicatura y materia impositiva y de auditoría. Acuerdan además que en lo que respecta a contabilidad y costos les compete, pero no exclusivamente.

Sin embargo, en la competencia "Registrar, medir y exponer información contable histórica y proyectada para todo tipo de organizaciones y unidades económicas", los estudiantes de Licenciatura en Economía encuestados en su mayoría la consideran una competencia específica pero no exclusiva; los encuestados de la carrera de Contador Público entienden que no les compete.

Los resultados arrojados por las respuestas de ambas carreras en esta parte de la pregunta sólo coinciden en que la competencia del manejo de técnicas cuantitativas y cualitativas le compete mas no exclusivamente.

En las otras tres competencias específicas: estructura y funcionamiento de mercados, análisis de indicadores y la producción de informes sobre impacto de políticas económicas, hay discordancia ya que los resultados de los estudiantes de Licenciatura en Economía consideran que no le compete al Licenciado en Administración; mientras que aquellos que dieron su respuesta y son estudiantes de Contador Público entienden que les compete más no exclusivamente. 


\section{REFERENCIAS BIBLIOGRÁFICAS:}

Oviedo, R. R., Leda, A. C., Rubino, M. C., Said, F. P., Schellas, T. (2021). "Percepción de los alumnos ingresantes a las carreras de Ciencias Económicas (UNR) Respecto a la Licenciatura en Administración". En: Actas Vigesimoquintas Jornadas "Investigaciones en la Facultad" de Ciencias Económicas y Estadística. Abril de 2021. ISSN № 1668-5008.

Oviedo, R. R., Racca, A. P., Suñer, M. N., Fior, M. y Ford, J. J. (2021). "Imagen de la Licenciatura en Administración en estudiantes de la carrera (UNR)". En: Actas Vigesimoquintas Jornadas "Investigaciones en la Facultad" de Ciencias Económicas y Estadística. Abril de 2021. ISSN № 1668-5008.

Universidad Nacional de Rosario - Facultad de Ciencias Económicas y Estadística. (2019) Resolución № 28982-C.D. "Plan de Estudios de la carrera de Licenciatura en Administración".

Universidad Nacional de Rosario - Facultad de Ciencias Económicas y Estadística. (2019) Resolución № 28981-C.D. "Plan de Estudios de la carrera de Licenciatura en Economía".

Universidad Nacional de Rosario - Facultad de Ciencias Económicas y Estadística. (2018) Resolución № 27817-C.D. "Plan de Estudios de la carrera de Contador Público". 